\title{
A Contrastive Study of Discourse Construction Features of Abstracts of Chinese and Foreign English Academic Papers From the Perspective of Contrastive Rhetoric
}

\author{
Yilun Yang (Corresponding author) \\ Nanjing Normal University, Nanjing, Jiangsu, China \\ E-mail: 820608774@qq.com \\ Liping Chen \\ Nanjing Normal University, Nanjing, Jiangsu, China
}

Received: August 15, 2020 Accepted: September 30, 2020 Published: October 20, 2020

doi:10.5296/ijl.v12i5.17854

URL: https://doi.org/10.5296/ijl.v12i5.17854

\begin{abstract}
Abstract is responsible for introducing research, attracting readers and other multiple functions, and has its own unique characteristics of discourse construction. Through objective analysis of the characteristics of discourse construction in abstracts published by Chinese and foreign scholars in international English journals, the accuracy of the expression of the articles can be increased. The author builds his own corpus and makes a contrastive analysis of the frequency, types and distribution of fuzzy restrictive language in each step of English abstracts in Chinese and foreign journals, and summarizes the unique short language types and textual functions of each step. The results show that the distribution trend of hedges in the fourth step of English abstracts in Chinese and foreign journals is generally the same, but there are significant differences in the types of use and the frequency of occurrence. Therefore, in English abstract writing, Chinese authors should properly use various types of rhetorical devices to enhance the diversity of language expression, so as to better obtain the recognition of readers.
\end{abstract}

Keywords: English abstract, Fuzzy restrictive language, Step, Contrastive analysis

\section{Introduction}

With the internationalization of Chinese science and technology and the increase of academic 
exchanges at home and abroad, more and more scholars publish British academic papers in foreign academic journals, and many kinds of English academic journals have been founded in China, especially the start of DEAP (Database of English for Academic purposes) academic English corpus construction project, which has promoted the academic development of domestic scholars and scholars' attention to academic English corpus. However, whether an academic thesis can be published in important journals at home and abroad, whether it can be recognized by its peers in the industry, in addition to the core research results shown in the paper itself, whether the writing level of academic papers can be recognized by international journals can not be ignored. However, it is difficult for Chinese scholars to write English, which affects the transmission and expression of academics. in addition to the mistakes in grammar and vocabulary spelling, the overall structure of the thesis is very different from that of native English researchers.

Although scholars at home and abroad have done some research on the abstracts and introductions of English academic papers from different aspects, most of them are based on a small number of corpus, so the credibility is low, because there is no corpus of Chinese English academic papers in China at present. In addition, most of the studies on English academic papers at home and abroad are the study of microcosmic language use and characteristics, and there are few studies from the macro discourse level, not to mention the contrastive study of discourse construction features of English academic journal papers from the perspective of functional grammar and textual contrastion. as for the systematic quantitative contrastive study based on corpus at the textual level of Chinese and foreign English academic journal papers, there are few systematic quantitative contrastive studies based on corpus at the discourse level of Chinese and foreign English academic journals. Therefore, the author believes that it is necessary to analyze the discourse construction of Chinese and foreign academic papers. The significance of this study is reflected in the following aspects: guided by the theory of systemic functional linguistics and genre analysis, through quantitative and qualitative analysis, this paper finds out the similarities and differences between Chinese and foreign academic papers and the reasons for the differences, and points out the shortcomings of Chinese scholars' journals, so it can guide the English academic writing of domestic scholars and help them to publish academic papers in important foreign journals. It is helpful to construct the internationalization of Chinese English academic thesis writing and improve the level of Chinese English academic thesis.

\section{Theoretical Framework}

\subsection{Contrastive Rhetoric}

Contrastive rhetoric is a branch of linguistics, which is a subject that compares two or more languages or linguistic subsystems in order to find out the similarities and differences. It mainly studies the problems in second language writing by comparing the differences between mother tongue and second language in rhetorical patterns, and contrastive rhetoric studies the similarities and differences of writing in different cultural backgrounds. Contrastive rhetoric scientists believe that different discourse expectations are the main reasons for the formation of different styles in cross-cultural writing. Second language 
authors may transform their first-language rhetorical strategies into a new second language environment before they fully understand the expectations of their second language listeners. The Cultural thinking Mode of Intercultural Education, published by R. Kaplan in 1966, studies the expression of the thinking mode and discourse structure of the first language in the second language. In his pioneering study of the paragraph organization of the thesis of the students who use English as a foreign language, Kaplan points out that the articles of the English language family are linear, while the rhetorical structure of the oriental language, including Chinese, is spiral. Oriental languages are asked, Latin languages and Russian articles like free themes. In the past 30 years, Kaplan has made a contrastive study of rhetoric from various angles and made a series of achievements, which has played an important role in foreign language teaching, especially in the development of English writing teaching. Hinds study the coherence of articles written by authors of different cultures. Kathpalia studies the differences in rhetorical structure, language model, vocabulary and grammar between international and local advertisements.

In the sixties and seventies of the 20th century, more and more linguists turned their studies to discourse, and the study of theme and rheme is increased in the area of functional grammar, and began to emphasize the social function of language and the information structure of discourse. In the 1980s, a large number of linguists promoted the combination of discourse analysis with other subjects and their application in teaching. Under the influence of discourse linguistics, the contrastive study of discourse rhetoric mainly focused on three aspects: cohesion, coherence and discourse pattern. Since the 1990s, with the development of discourse linguistics in China, many contrastive studies have emerged from the perspectives of cohesion, coherence and discourse patterns. However, few people specialize in rhetorical contrastive research, and the depth of related research needs to be further improved.

\subsection{Fuzzy Restrictive Language}

In 1996, Halliday put forward that hedges and boosters can express both conceptual and interpersonal information. Their use indicates that the author is in the paper, can more accurately convey the information of the paper, and better reflect the dialogue with the readers. in the same year, Low called this finite element in academic papers as lexical Invisibility hypothesis, in fact. This kind of participation expression of the author is one of the core problems of rhetorical strategies in the writing of academic papers.

Fuzzy linguistics is a new subject formed by the combination of fuzzy set theory and modern linguistics. It uses the basic theory of fuzzy set theory and modern linguistics to analyze the fuzziness of language. After Wei Zadeh (1969) put forward the concept of fuzzy and fuzzy set theory, Lakoff 1972) put forward the term "hedges" for the first time. The linguistic study of hedges reflects a very important pragmatic value and attracts scholars from all over the world to study them in depth. Prince et al. (1982) analyzed the hedges in doctor's language and proposed a classification model of hedges. Many researchers (Chafe, Meyer, Low, Biber, etc) noticed that hedges have modified, qualified concepts or propositions, avoid saying things too dead, make speech express spiritual words, have room for manoeuvre, and have pragmatic functions such as disguising and concealing personal views. Salager-Myers (1994) holds that 
the use of hedges is a mental attitude. Hyland (1998) discusses the pragmatic functions of hedges from different angles which are beneficial to the accuracy of the content expression of scientific and technological papers, the advantages of the author and the readers. In academic discourse, it is the main rhetorical strategy used by the author to ease the power of criticism or potential threats from peers (Myers, 1989). Therefore, it is considered to be the most common, typical and valuable language.

Contrary to the pragmatic function of hedges, the function of boosters is to strengthen the expression of language, to convince people to express their views, and to reduce or dispel each other's doubts. Chafe (1985) once divided the boosters into two categories: one is that the knowledge is related to the reader's body and the other is the knowledge shared by both sides; the other is that the author has full faith in the information and there is no doubt about it. In short, the use of boosters reflects a certain interpersonal function, attracts readers' attention to a specific discussion, and encourages readers to dispute the views they state in order to obtain the maximum attention of readers. Sometimes, an idea in an academic article or paper can get the reader's attention, even if it is controversial, it also explains the value of the academic paper from one side.

\subsection{Genre and Genre Analysis}

At first, this concept was usually used in the study of literature and rhetoric, referring to written chapters, and then applied to daily dialogue and political speeches. Genre analysis is mainly composed of two schools, the American traditional school represented by John Swales and the Australian school represented by Jim Martin. The American traditional school holds that each genre is an example of the successful use of conventional language and discourse resources to achieve a specific communicative purpose. This definition is often cited by scholars, who use genre as a tool to analyze and teach spoken and written languages used by non-native English speakers in academic and professional environments. According to the formal characteristics and communicative purpose of the genre, scholars divide the genre into oral discourse and written text.

Among them, Swales combines linguistic and sociological factors well in the definition of genre, but ignores psychological factors. He believes that genre is composed of a series of communicative events, and communicative members have a common communicative purpose. Swales points out that the introduction of academic papers consists of three steps, and the structure of language steps is a model in which different changes can be made according to the degree of genre idiom or custom. From the point of view of academic English, genre is composed of a series of language steps, each step can be taught to beginners of a particular genre as a chapter section, and many scholars use structural step analysis to describe the structural pattern of genre.

In a word, the analysis of these practical and professional genre structures provides guidance and reference for the analysis of discourse genre structure of English academic papers. 


\section{Research Process}

\subsection{Corpus Collection}

This study will use a comparative method to collect 50 abstracts of papers in domestic core journals in 2010 (according to the catalogue of core journals of Peking University in 2008). The corpus of English abstracts collected from the United States in 2010 is the true natural corpus (according to the top 50 journals with the top 50 influencing factors in 2009). The English abstract corpus (CRC) (written by Chinese authors, and the English abstract corpus (ARC) (written by American authors, are established with 7623 English words and 6129 English words, respectively.

Based on the self-built corpus, the quantitative and qualitative studies are carried out, and the textual laws and characteristics of English and Chinese academic papers of the same genre are expected to be obtained.

\subsection{Analytical Methods}

The author will adopt the methods of quantitative statistics and qualitative analysis. This paper makes tagging and discourse analysis of English academic papers from the aspects of the length of the abstract, the number of paragraphs, the number of sentences, the pattern of language steps, the frequency and the type of short words of the two kinds of modifiers.

The main contents are as follows:

(1) Stylistic analysis is a kind of genre analysis

Genre analysis is a study of language use in a specific language environment, which will vary according to the purpose of communication. Genre analysis is not only an applied linguistics, but also a method in comprehensive sociology, psychology and linguistic text analysis. The author intends to use Swales' step model (Introduction, Methods, Results and Discussion), uses PowerGREP to extract four language steps from 100 English abstracts of Chinese and foreign papers). Eight independent corpus are built: Chinese Introduction Corpus (CI), Foreign Introduction Corpus (FI), Chinese Method Corpus (CM), Foreign Method Corpus (FM), Chinese Result Corpus (CR), Foreign Result Corpus (FR), Chinese Discussion Corpus (CD) and Foreign Discussion Corpus (FD), in order to make a comparative analysis of the characteristics of each step.

(2) Comparative analysis

This paper makes a statistical analysis of the distribution of hedges in each step of the thesis. SPSS is used to retrieve this type of hedges respectively, and the frequency of various types of hedges in each language step is extracted, so that we can make a comparative analysis.

\subsection{Key Issues to Be Addressed}

The main contents are as follows:

(1) What are the similarities and differences in the use of modifiers in abstracts of Chinese and English academic papers? And what is the relationship between these similarities and 
differences and social and cultural differences?

(2) Compared with the abstracts of foreign academic papers, what are the typical short language types and textual functions in the abstracts of domestic academic papers? What are the differences in phrase patterns and discourse strategies in abstracts at home and abroad? What are the characteristics of discourse strategies in the typical short language patterns used by scholars at home and abroad?

\section{Results and Discussion}

Structurally speaking, the general requirements of English academic journal papers are at least six basic parts: abstract, introduction, material and method, research results, discussion and conclusion. This paper mainly studies the discourse construction of abstract, because abstracts are more regular and reflect the author's discourse construction ideas and ideas, as well as the practice of thesis writing.

Abstract part is the most important part of the paper, on the basis of the International Standardization Organization (ISO), it is a brief and accurate expression of the content of the document, which is independent and self-contained. First of all, as a relatively independent subtext of the study, this paper briefly introduces the research object, research methods and main research results. In addition, it helps readers who want to read the entire article to familiarize them with the context of the article in advance. Second, help readers screen the article and decide whether to improve the reading of the whole chapter. Third, it serves as an index tool to provide key information for scholars and editors. Moreover, the abstract provides help for the reviewers to decide whether to accept the article or not. This requires the author to summarize the purpose, main research contents, research methods and conclusions of this paper in the most concise language and to enable readers to master the main purpose of the literature quickly.

So far, there are few empirical studies on the rhetorical structure of abstracts, and only the rhetorical structure of reported excerpts has been recognized and described. Many researchers have focused on verb tenses, voice, modal verbs and other linguistic features. In recent years, some scholars have found that the use of verb tenses and modal verbs is closely related to the rhetorical function of abstract steps.

The author analyzes and discusses the abstracts of Chinese and foreign academic papers from the following angles:

\subsection{A Comparative Study of Chinese and Foreign Academic Abstracts by Comparing the Distribution of Modifier}

Hyland (2000) classifies the restrictive components used to modify the author's statement in academic discourse into hedges and boosters. The former refers to the restrictive modifiers used to express uncertainty or lack of assurance, as a polite means to cushion the invasion of the "face" of other scientists and enhance the consistency with the readers. The latter indicates that the author believes in the views discussed or shows that the author personally participates in the experiment or is responsible to the readers. 
In the study of hedges in China, the hedges in this paper are divided into s categories: 1) hedges (could, might, may, would, should, etc.); 2) hedges in the category of table quantity and degree (some, many, somewhat, approximately, a large amount of, typically, frequently, rarely, often, essentially, roughly, to some extent, etc.); 3) hedges that express possibility(likely, possibly, probably, presumably, etc.) 4) the hedges that express cognitive category (suggest, seem to, appear to, indicate, propose,imply etc.): 5)hedges express the author's personal point of view or resonates with the reader (believe, to our knowledge, it is our viewpoint etc. or Surprisingly, Interestingly, etc.).

Table 1 shows that the distribution trend of hedges in the four steps of English abstracts in Chinese and American journals is generally the same. Hedges are mainly concentrated in the conclusion part, and their frequency of use is much higher than that in the other three parts, because the authors of the conclusion part mainly state their own research results, evaluate them and put forward their own views. The second part is the result part, which describes the data and findings obtained in the experiment. The acquisition of these data is affected by various factors. It is very difficult to achieve accuracy, they are only to some extent accurate, so the author needs to use some type of hedges to express their incompleteness or uncertainty. The method part mainly describes the specific methods and operation steps used in the experiment, which are conclusive facts, so hedges are rarely used. The biggest difference in the frequency of use is in the introduction part, where the frequency of use in the foreign academic papers is 4.57 percentage points higher than in China. Because the introduction part of the abstract of the Chinese paper likes to use infinitesimal phrases best, and the most favorite thing in the foreign abstract is to use complete sentences to make their expressions more accurate.

Table1. Frequency of hedges per 1000 words in each move

\begin{tabular}{lllllllll}
\hline type & CI & FI & CM & FM & CR & FR & CD & FD \\
\hline Wish & 0.59 & 2.88 & 0.37 & 0.48 & 1.25 & 0.77 & 15.58 & 11.93 \\
\hline Quantity degree & 0.08 & 1.95 & 0.06 & 0.34 & 0.30 & 1.28 & 1.45 & 2.01 \\
\hline possibility & 0.01 & 0.07 & 0.00 & 0.07 & 0.10 & 1.45 & 0.56 & 1.93 \\
\hline cognition & 0.09 & 0.28 & 0.01 & 0.08 & 0.01 & 0.16 & 1.07 & 3.12 \\
\hline Personal opinion, cause & 0.02 & 0.18 & 0.01 & 0.01 & 0.00 & 0.00 & 0.01 & 0.04 \\
\hline In total & 0.79 & 5.36 & 0.45 & 0.98 & 1.66 & 3.66 & 18.67 & 19.03 \\
\hline
\end{tabular}

The reasons for the differences: the macro structures of foreign and Chinese academic thesis abstracts show that foreign and Chinese academic texts are different oriented, potential and abstract structures, which are influenced by the culture recognized by the communicators. It also reflects the different social and cultural concepts in the subconscious mind of Chinese and western scholars. In English community countries, in order to make the thesis acceptable, scholars must adopt some rhetorical strategies to declare the innovation of their thesis research, so the use of boosters in English abstracts is more than that in Chinese abstracts. In China, because of the differences between Chinese and foreign cultural customs, scholars' attitude towards their own scientific research results is modest and cautious, so many scholars use hedges in abstracts. In addition, the difference is determined by two factors: the education 
system and intertextuality. The educational system of the foreign countries emphasizes the teaching of skills, and the writing materials are many and will elaborate on the rules of writing in detail, and provide specific models for imitation: while the teaching system of Chinese education in English emphasizes content, light form, emphasis on explanation of ideological content, light analysis of article structure framework, so that students know less about some common stylistic forms. With regard to intertextuality, Williams argues that "the output or perceived acceptance of a given modifiers depends on the reader's mastery of other texts." In other words, each text is not independent, but is related to some of the original texts, which together constitute the cultural traditional development model of the region. Some cognitive schemas will naturally be projected to the new text, which will be deeply rooted in the hearts of every member of the culture. This is obvious to those who learn a foreign language: nevertheless, we need to be aware of this difference and pay attention to it in academic discourse writing.

\subsection{A Comparative Study of Chinese and Foreign Academic Abstracts in Terms of Short Language Types and Textual Functions}

Academic papers not only have their unique discourse structure, but also contain phrase sequences and short language patterns. These linguistic entities embody the idiom principle and phraseological tendeney of language communication. The reasonable and effective use of the phrase patterns in academic discourse is an important reference to judge the academic communicative competence of the author of the thesis, and it is also a sign of the maturity of the researcher in his academic discourse community. For Chinese scholars, how to use academic phrase forms naturally and appropriately in different discourse structures is a question worthy of consideration. Therefore, the author manually recognizes and examines the moves of abstracts in corpus. Finally, by comparing the phrase patterns and textual functions of each move, this paper focuses on the reasons for the differences in the phrase patterns, so as to provide a new perspective for domestic scholars in scientific research and English academic writing teaching.

First, in the I language step, in order to highlight the previous research results, Chinese and foreign scholars use the phraseological tendency "QUA+research has/studies have+V" to summarize or evaluate the previous research. However, in this type, domestic scholars often use "dedicated to, achieved" and other verbs to evaluate the research results, while foreign scholars use "show, demonstrated" and other verbs to illustrate the findings of existing studies. In addition, the quantitative word qualification research commonly used by domestic scholars shows that there are a large number of research literature on a certain topic. Foreign scholars use adjectives to limit previous studies, or use the negative quantifier "little, no" to show that there is not a large number of related studies. The differences in the use of co-expression show that domestic scholars use to highlight the previous research results, while foreign scholars tend to directly point out the shortcomings of previous studies.

In view of the insufficient attention paid to the research topics, domestic scholars often use the negative expressions in "ADVneg+ADJ+analysis of+NPtopic", while foreign scholars often use "Vproblem+the question of+NPtopic" or the qustion of+ "NPtopic+Vproblem". 
The evaluation attitude of domestic scholars to the previous research is mild, which tends to attribute the problem to the limitation of attention; foreign scholars clearly point out the unresolved problems and establish a clear research space.

In order to state the significance and purpose of the study, domestic scholars use "V+understanding of..". Foreign scholars use the phrase patterns "V / an+ADJ+approach to" to explain the research perspective or research methods.

Second, M language move emphasizes the reasonableness of the research method and state how the research is carried out. Domestic scholars often use the phrase patterns of "D+dissertation / study+VP", often accompanied by "based on and from the perspective of "and other connected phrases to explain the research framework; while foreign scholars often use connection phrases to explain the analysis methods and data sources, such as "Through a series of case studies.." Domestic scholars tend to use verb passive structure to explain data acquisition and data processing, such as "The data+BE collcted from.." The use of passive structure shows that the author of the paper has always maintained a neutral and objective academic attitude, while foreign scholars often use active structure to explain data collection information, such as "V+data".

Third, in the R language step, the domestic authors tend to report objectively the results of the whole study or a specific experiment, and rarely use the short language type, "It BE found tha...." with self-referential, "results of+Nstudy+V...". Foreign scholars often use "I" or "we" to co-appear with reported verbs, such as "I / We+ V", paying attention to the interaction with readers. On the other hand, domestic scholars focus on description and evaluation, foreign scholars tend to report on the differences or causality between the objects of study.

Fourth, in the D step, domestic scholars tend to use nouns referring to the results of macro-research to summarize and evaluate the study as a whole, and the tone is more objective. Foreign scholars use "I" or "we" to refer to the author of the thesis at the end of the abstract text, indicating that the author of the thesis is responsible for the statement of the conclusion of the study. In addition, when discussing the enlightenment and significance, the abstract of the internal paper has a large capacity of information, and the common phrase patterns are commonly used by domestic scholars, such as "implications of $+\mathrm{N}$ " and "implications for $+\mathrm{N}$ ", to specify the research enlightenment or put forward the research limitations with "Limitations of the study were.../recomendations for future studies were finally+V-en", while foreign scholars tend to mention it once and for all, and only observe the short language type $\mathrm{N}+$ for future research that puts forward suggestions for future research.

In summary, academic research in China tends to be regarded as a process of producing new academic discoveries and transmitting new academic information through the inheritance, verification or re-interpretation of the theory. Foreign academic papers tend to regard academic research as a process of solving research problems and creating new knowledge systems through challenges to specific problems, re-interpretation and reconstruction of concepts.

\section{Conclusion}

In this paper, the corpus of English academic papers is established strictly according to the 
requirements of systematic sampling and hierarchical sampling, and qualitative and quantitative analysis methods are used to lay a solid data foundation for corpus analysis and data processing. Moreover, this study makes a contrastive study on the frequency, distribution and types of hedges in abstracts of Chinese and foreign English academic papers, and summarizes and analyzes the short language patterns and textual functions of each step. The results show that as far as the frequency of five types of hedges is concerned, Chinese scholars overuse hedges in the category of wish, while the other four types use hedges much lower than those of American scholars; as far as their distribution is concerned, similarities and differences coexist, and hedges are mainly concentrated in the conclusion and result parts, and the use of hedges in the target step is the most different. It can also be seen from the data that American scholars can apply various types of hedges to different language steps more flexibly, while the use of hedges by Chinese scholars is relatively single. There are many reasons for the differences in the use of hedges between Chinese and American authors. On the one hand, they may be related to the English proficiency of domestic authors, and the limited knowledge of English is not comparable to that of native English speakers. For domestic scholars, it is still difficult to express their ideas accurately in English as flexibly as native speakers of English. Secondly, the negative transfer of the native language culture of the domestic authors can not be ignored. In addition, a considerable proportion of the abstracts of English academic papers written by the domestic authors are not standardized and fail to meet the requirements of the writing standards of the English abstracts.

Because of its unique function, it is usually considered to be the most important part of professional papers, which has the characteristics of objective and concise. However, it is still a difficult problem for Chinese scholars to organize the abstract of a scientific paper effectively. Therefore, the purpose of this study is to improve the structure of abstracts in English academic journals from the surface and deep structures of language, and to find out the similarities and differences between abstracts written by Chinese and foreign scholars, so as to provide some skills for compiling a well-organized abstract.

Chinese scholars should improve their English level, improve their writing ability of standard English abstracts, and play the role of hedges in English abstract writing to make the language standard and appropriate, so as to realize the academic influence of the thesis.

\section{References}

Brown, P., \& Levinson, S. (1978). Universals in Language Usage: Politeness Phenomena. In E. N. Goody (Ed.), Questions and Politeness: Strategies in Social Interaction (pp. 56-289). Cambridge: Cambridge University Press.

Chen, L. P. (2007). A Summary of the study of English discourse structure tagging. Foreign Languages and Foreign Language Teaching, 16(7), 9-10.

Feng, Y., \& Zhou, R. (2007). Investigation and Analysis of hedges in Abstracts of academic papers. Foreign Language and Literature, 24(2), 108-112.

Hu, G., \& Cao, F. (2011). Hedging and boosting in abstracts of applied linguistics articles: A comparative study of English and Chinese medium journals. Journal of Pragmatics, 2011(43), 2795-2809. 


\section{Macrothink}

International Journal of Linguistics

ISSN 1948-5425

2020, Vol. 12, No. 5

$\mathrm{Hu}, \mathrm{X}$. (2015). A contrastive study on the features of step lexical chunks in Chinese and foreign abstracts of science and technology. Modern Foreign Language, (6), 813-822.

Hyland, K. (1998). Hedging in scientific research articles (pp. 116-249). Amsterdam and Philadelphia: John Benjamins Publishing Company.

Hyland, K. (2000). Disciplinary discourse: social interactions in academic writing. London: Longman.

Hyland, K. (2005). Metadiscourse: exploring interaction in writing. London: Continuum.

Ju, Y. M. (2004). Genre Analysis and Abstracts of English and Chinese academic papers. Foreign Language Teaching, (2), 32-35.

Martin-Martin, P. (2008). The Mitigation of Scientific Claims in Research Papers: A Comparative Study. International Journal of English Studies, 8(20), 133-152.

Rimrott, A. (2007). The discourse structure of research article abstracts: A Rhetorical Structure Theory (RST) analysis. The Proceedings of the 22nd North West Linguistics.

Varttala, T. (2001). Hedging in scientifically oriented discourse: exploring variation according to discipline and intended audience. Tampere: University of Tampere.

Van Bonn, S., \& Swales, J. M. (2007). English and French journal abstracts in the language sciences: Three exploratory studies. Journal of English for Academic Purposes, 6, 93-108.

Wang, L. Y., Civilization, Y., Wang, L. Z., et al.. (2011). Contrastive Analysis of English Abstracts of Chinese and American Medical papers. Science and Technology and Publishing, (11), 78-82.

Wang, H. L. (2005). An Analysis of hedges in English academic papers of Chinese-speaking second language Learner. Dalian: Dalian Maritime University.

Wang, W. (1994). A Review of Rhetorical Structure Theory (First part). Foreign Linguistics, 1994(4), 8-13.

Wu, T. P. (1999). Fuzzy linguistics (pp. 71-82). Shanghai: Shanghai Foreign Language Education Press.

Zhao, Y. L. (1999). Hedges in English Scientific and Technological Style. Foreign Language and Foreign Language Teaching, 1999(9), 15-17.

Zhang, H. (2005). Interpersonal Function of Hedges in EST Texts. Journal of He Fei University of Technology (Social Science Edition), 2005(5), 85-87.

\section{Copyrights}

Copyright for this article is retained by the author(s), with first publication rights granted to the journal.

This is an open-access article distributed under the terms and conditions of the Creative Commons Attribution license (http://creativecommons.org/licenses/by/4.0/) 